\title{
Flavor SU(3) breaking effects in the chiral unitary model for meson-baryon scatterings
}

\author{
T. Hyodo, ${ }^{1}$ S. I. Nam, ${ }^{1,2}$ D. Jido, ${ }^{1}$ and A. Hosaka ${ }^{1}$ \\ ${ }^{1}$ Research Center for Nuclear Physics (RCNP), Ibaraki, Osaka 567-0047, Japan \\ ${ }^{2}$ Department of Physics, Pusan National University, Pusan 609-735, Korea
}

(Dated: October 28, 2018)

\begin{abstract}
We examine flavor $\mathrm{SU}(3)$ breaking effects on meson-baryon scattering amplitudes in the chiral unitary model. It turns out that the SU(3) breaking, which appears in the leading quark mass term in the chiral expansion, can not explain the channel dependence of the subtraction parameters of the model, which are crucial to reproduce the observed scattering amplitudes and resonance properties.
\end{abstract}

PACS numbers: 12.39.Fe, 11.80.Gw, 14.20.Gk, 14.20.Jn, 11.30.Hv

Keywords: chiral unitary approach, meson-baryon scatterings, flavor SU(3) breaking

Properties of baryonic excited states are investigated with great interest both theoretically and experimentally. Recently, the chiral unitary model has been successfully applied to this problem, especially to the first excited states of negative parity $\left(J^{P}=1 / 2^{-}\right)$such as $\Lambda(1405)$ and $N(1535)$ [1, 2, 3, 4, 5, 6, 7]. In this method, based on the leading order interactions of the chiral Lagrangian and the unitarization of the S-matrix, the baryon resonances are dynamically generated as quasi-bound states of ground state mesons and baryons. It reveals the importance of chiral dynamics not only in the threshold but also in the resonance energy region.

In the chiral unitary model for the meson-baryon scattering, we consider the coupled channel scatterings of the octet mesons and baryons. Imposing the unitarity condition on the scattering amplitudes $T_{i j}$ in the $N / D$ method, we obtain the scattering equation in the matrix form Refs. [3, 8]:

$$
T_{i j}=V_{i j}+V_{i k} G_{k} T_{k j},
$$

where $V_{i j}$ denotes the elementary tree level interaction derived from the chiral Lagrangian. This equation can be solved algebraically. The loop integral $G_{i}$ is the fundamental building block in the chiral unitary model and are regularized by the dimensional regularization;

$$
\begin{aligned}
G_{i}(\sqrt{s})= & i \int \frac{d^{4} q}{(2 \pi)^{4}} \frac{2 M_{i}}{(P-q)^{2}-M_{i}^{2}+i \epsilon} \frac{1}{q^{2}-m_{i}^{2}+i \epsilon} \\
= & \frac{2 M_{i}}{(4 \pi)^{2}}\left[a_{i}(\mu)+\ln \frac{M_{i}^{2}}{\mu^{2}}+\frac{m_{i}^{2}-M_{i}^{2}+s}{2 s} \ln \frac{m_{i}^{2}}{M_{i}^{2}}\right. \\
& \left.+\frac{\bar{q}_{i}}{\sqrt{s}}\left(\operatorname{Ln}_{+-}+\operatorname{Ln}_{++}-\operatorname{Ln}_{-+}-\operatorname{Ln}_{--}\right)\right],
\end{aligned}
$$

with $\left.\operatorname{Ln}_{ \pm \pm} \equiv \ln \left( \pm s \pm\left(M_{i}^{2}-m_{i}^{2}\right)+2 \sqrt{s} \bar{q}_{i}\right)\right)$, the masses of baryon and meson $M_{i}$ and $m_{i}$, the three-momentum of the meson $\bar{q}_{i}$, the total energy in the center of mass system $\sqrt{s}$ and the regularization scale $\mu$. In the present calculation, we follow the method shown in refs. 5, 6, 9], and calculate only the $s$-wave amplitudes since the contributions from the $p$-wave (and higher partial waves) are much less important in energies considered here [10].

In actual calculations, it is necessary to determine the renomalization constants ( $a_{i}$ 's) in Eq.(2) so as to repro-

\begin{tabular}{ccccccc}
\hline \hline$S=-1$ & $\bar{K} N$ & $\pi \Sigma$ & $\pi \Lambda$ & $\eta \Lambda$ & $\eta \Sigma$ & $K \Xi$ \\
\hline$a_{i}$ & -1.84 & -2.00 & -1.83 & -2.25 & -2.38 & -2.67 \\
\hline \hline \hline & & & & & & \\
\hline \hline$S=0$ & $\pi N$ & & $\eta N$ & $K \Lambda$ & $K \Sigma$ \\
\hline$a_{i}$ & 0.711 & -1.09 & 0.311 & -4.09 \\
\hline \hline
\end{tabular}

TABLE I: Channel dependent subtraction constants $a_{i}$ obtained in Refs. 5, 6] with $\mu=630 \mathrm{MeV}$.

duce experimental data. The constants $a_{i}$ are equivalent to the subtraction constants in the dispersion theory formulation [8] and, in fact, are free parameters of the model. As a consequence, they have depended very strongly on scattering channels, as shown in Table \

In this work, we investigate whether such channel dependence of the subtraction constants could be explained by the $\mathrm{SU}(3)$ breaking terms of the chiral perturbation theory. By doing this, we expect that the free parameters of $a_{i}$ 's could be controlled with suitable physics ground, namely the $\mathrm{SU}(3)$ breaking terms, in order to extend this model to various channels with predictive power. Here we keep using just one subtraction constant $a$ commonly in all channels to regularize the loop function $G_{i}$.

The use of only one subtraction constant is justified in the $\mathrm{SU}(3)$ symmetric limit $[9,11]$. Under the flavor $\mathrm{SU}(3)$ symmetry, the scattering amplitude should be expressed as a diagonal matrix in the $\mathrm{SU}(3)$ basis $(1,8, \cdots)$, which is transformed from the particle basis $(\pi N, \eta N, \cdots)$ by a fixed unitary matrix given by the $\mathrm{SU}(3)$ Clebsch-Gordan coefficients. Each components of the amplitude $T(D)$ separately satisfies the scattering equation like Eq.(2) in each irreducible representation $D$. Therefore, on one hand, the function $G$ represented in a matrix form becomes a diagonal matrix in the $\mathrm{SU}(3)$ basis. On the other hand, since the $G$ function is given as a loop integral as shown in Eq. (2), it is also diagonal in the particle basis. Therefore the subtraction constants $a_{i}$ 's are components of a diagonal matrix both in the $\mathrm{SU}(3)$ and particle bases. Such a matrix for the subtraction constants should be proportional to unity. Hence, it is concluded that there is only one subtraction constant $a$ in the $\mathrm{SU}(3)$ limit.

Now let us show the Lagrangian with the flavor SU(3) 
breaking terms, which we use in the present work. The $\mathrm{SU}(3)$ breaking appears as the quark mass terms in the chiral expansion:

$$
\begin{aligned}
\mathcal{L}_{S B}= & -\frac{Z_{0}}{2} \operatorname{Tr}\left(d_{m} \bar{B}\left\{\xi \mathbf{m} \xi+\xi^{\dagger} \mathbf{m} \xi^{\dagger}, B\right\}\right. \\
& \left.+f_{m} \bar{B}\left[\xi \mathbf{m} \xi+\xi^{\dagger} \mathbf{m} \xi^{\dagger}, B\right]\right) \\
& -\frac{Z_{1}}{2} \operatorname{Tr}(\bar{B} B) \operatorname{Tr}\left(\mathbf{m} U+U^{\dagger} \mathbf{m}\right) .
\end{aligned}
$$

where $f_{m}+d_{m}=1$. Here we employ the standard notation [12]: $\xi(\Phi)=\exp \{i \Phi / \sqrt{2} f\}$ and $U(\Phi)=\xi^{2}$. The $3 \times 3$ matrices $B$ and $\Phi$ represent the baryon and meson fields. At this stage, we introduce one meson decay constant $f$, which is taken as an averaged value $f=1.15 f_{\pi}$ with $f_{\pi}=93 \mathrm{MeV}$. The quark mass matrix is defined as $\mathbf{m}=\operatorname{diag}\left(\hat{m}, \hat{m}, m_{s}\right)$ with isospin symmetry, $m_{u}=m_{d} \equiv \hat{m}$. The parameters $Z_{0}, Z_{1}, f_{m} / d_{m}$ can be determined by the baryon masses and the $\pi N$ sigma term, and therefore we have no new free parameters. Here we have $Z_{0}=0.528, Z_{1}=1.56$ and $f_{m} / d_{m}=-0.31$ with $m_{s} / \hat{m}=26$, which are determined in chiral perturbation theory for meson masses. The terms in Eq. (3) are of order $\mathcal{O}\left(p^{2}\right)$, based on the Gell-Mann-Oakes-Renner relation [13], which implies $m_{q} \propto m_{\pi}^{2}$. There are other chirally symmetric terms of order $\mathcal{O}\left(p^{2}\right)$. Here we do not take into account these terms, since we concentrate on the effects of the flavor $\mathrm{SU}(3)$ breaking.

Let us show the numerical results of the $\bar{K} N$ induced scatterings. We use a single subtraction constant $a$, and compare the results with and without the $\mathrm{SU}(3)$ breaking terms. In each case, the subtraction constant is determined by fitting threshold branching ratios 14, 15]:

$$
\begin{aligned}
\gamma & =\frac{\Gamma\left(K^{-} p \rightarrow \pi^{+} \Sigma^{-}\right)}{\Gamma\left(K^{-} p \rightarrow \pi^{-} \Sigma^{+}\right)} \sim 2.36 \pm 0.04, \\
R_{c} & =\frac{\Gamma\left(K^{-} p \rightarrow \text { charged particles }\right)}{\Gamma\left(K^{-} p \rightarrow \text { all }\right)} \sim 0.664 \pm 0.011 \\
R_{n} & =\frac{\Gamma\left(K^{-} p \rightarrow \pi^{0} \Lambda\right)}{\Gamma\left(K^{-} p \rightarrow \text { neutral particles }\right)} \sim 0.189 \pm 0.015
\end{aligned}
$$

Without the symmetry breaking terms, we find the optimal value $a=-1.96(\mathrm{~A})$. Now including the symmetry breaking term, the optimal value takes $a=-1.59$ (B). The calculated threshold values are presented in Table III From the table, we see that the agreement with data is improved by including the symmetry breaking effect. Note that this improvement is achieved without new free parameters.

Using these optimal values, we calculate the cross sections of $K^{-} p \rightarrow$ (various channels) and plot them in Fig. 1 Results of (A) are shown by dotted lines and those of (B) by dash-dotted lines. For (A), the agreement with data is still good, which is the well known result of the chiral unitary model $[1,[3,8]$. Originally the subtraction constants in the $S=-1$ channel are not very

\begin{tabular}{cccc}
\hline \hline & $\gamma$ & $R_{c}$ & $R_{n}$ \\
\hline experiment & $2.36 \pm 0.04$ & $0.664 \pm 0.011$ & $0.189 \pm 0.015$ \\
\hline (A) & 1.80 & 0.624 & 0.225 \\
$(\mathrm{~B})$ & 2.19 & 0.623 & 0.179 \\
$(\mathrm{C})$ & 2.35 & 0.626 & 0.172 \\
\hline \hline
\end{tabular}

TABLE II: Threshold branching ratios calculated by using $a=-1.96$ without the $\mathrm{SU}(3)$ breaking interaction (A), $a=$ -1.59 with the $\mathrm{SU}(3)$ breaking interaction (B), $a=-1.68$ with the SU(3) breaking interaction and the physical $f(\mathrm{C})$. Experimental values are take from Refs. [14, 15].
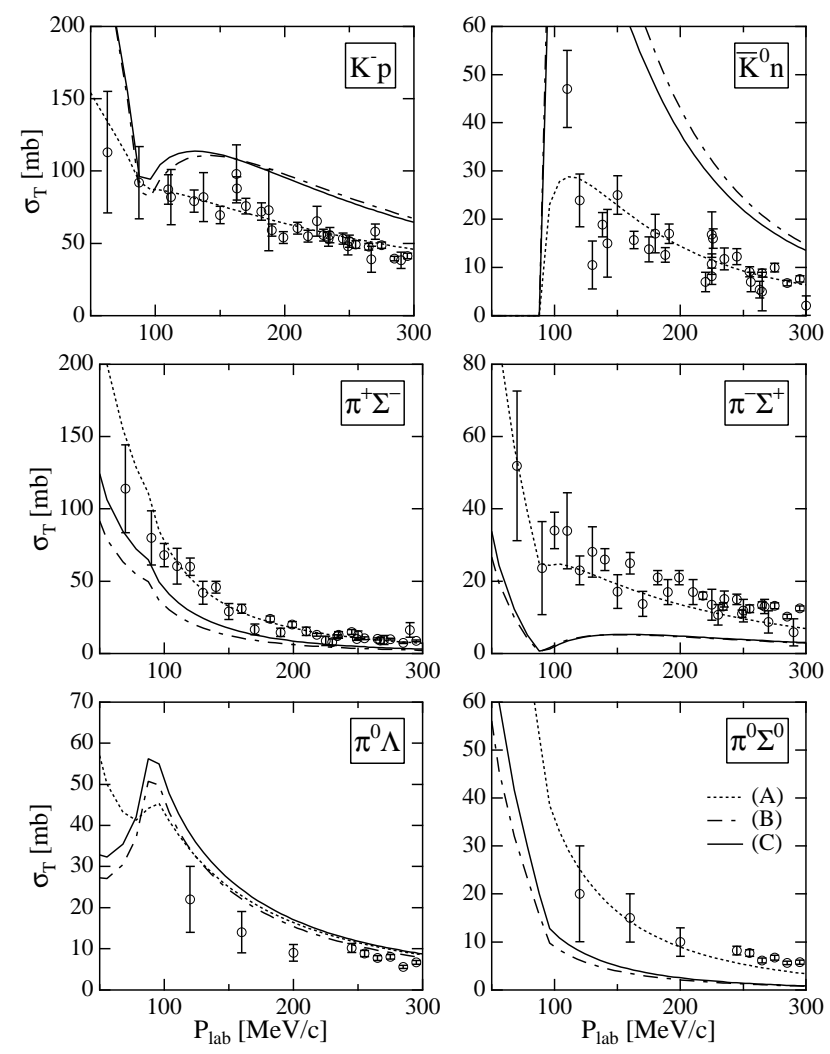

FIG. 1: Total cross sections of $K^{-} p$ scatterings $(S=-1)$ as functions of $P_{\mathrm{lab}}$, the three-momentum of initial $K^{-}$in the laboratory frame. Dotted lines show the results with $a=$ -1.96 without $\mathrm{SU}(3)$ breaking (A), dash-dotted lines show the results including the $\mathrm{SU}(3)$ breaking with $a=-1.59(\mathrm{~B})$, and solid lines show the results including the $\mathrm{SU}(3)$ breaking and the physical $f$ with $a=-1.68(\mathrm{C})$. Open circles with error bars are experimental data taken from Refs. [16, 17, 18, 19, 20, 21, 22, 23, 24].

much dependent on the channels and take values around $a_{i} \sim-2$ as shown in Table \ Now including the symmetry breaking terms (B), we find that agreement with data becomes worse (dash-dotted lines), contrary to our expectation, although the threshold branching ratios are better reproduced. 


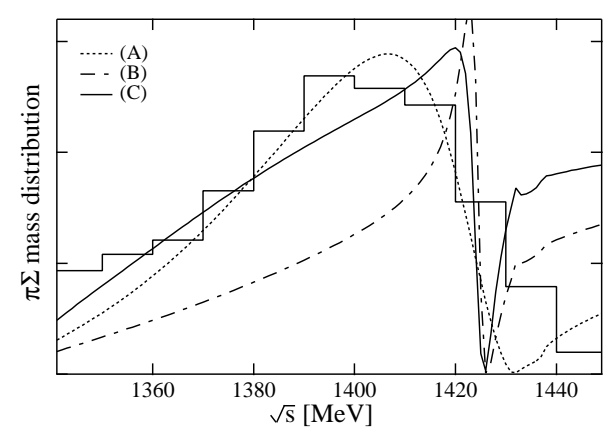

FIG. 2: Mass distributions of the $\pi \Sigma$ channel with $I=0$. Dotted line shows the result with $a=-1.96$ (A), dash-dotted line shows the result including the $\mathrm{SU}(3)$ breaking with $a=$ -1.59 (B), and solid line shows the result including the $\mathrm{SU}(3)$ breaking and the physical $f$ with $a=-1.68(\mathrm{C})$. Histogram are experimental data taken from Ref. [25].

In Fig. 2 we show the $\pi \Sigma$ mass distribution, in order to investigate the $\Lambda(1405)$ resonance. For $(\mathrm{A})$ we obtained the dotted curve which agrees well with experimental data. If we include the symmetry breaking terms (B), once again, the agreement becomes worse as shown by dash-dotted line. A sharp peak is pronounced around $\sqrt{s}=1420 \mathrm{MeV}$, in obvious contradiction with the observed spectrum.

We also perform calculations with the inclusion of another source of the $\mathrm{SU}(3)$ flavor breaking, that is, the meson decay constants. We use the empirical values of the decay constants: $f_{\pi}=93 \mathrm{MeV}, f_{K}=1.22 f_{\pi}, f_{\eta}=$ $1.3 f_{\pi}$. The optimal value of the subtraction constant $a$ in this case is $a=-1.68$ (C) to reproduce the threshold ratios. The results are shown in Figs 1, 2] with the solid lines. While the inclusion of the $\mathrm{SU}(3)$ breaking on the meson decay constants does not make drastic improvement in the total cross sections of the $K^{-} p$ scatterings as shown in Fig 1 the shape of the peak in the $\pi \Sigma$ mass distribution becomes milder. However, the improvement is not enough to reproduce the experimental spectra.

We perform similar analyses for the $\pi N$ scattering for the $S=0$ channel. At first we use the common subtraction constant $a=-1.96$ obtained in the $S=-1$ channel without the symmetry breaking, since it reproduces the $\Lambda(1405)$ property well and we want to check the SU(3) flavor symmetry. Then the attractive force between the mesons and baryons is so strong that an unexpected resonance has been generated at around $\sqrt{s} \simeq 1250 \mathrm{MeV}$. Therefore we choose the values of $a$ for $S=0$ by fitting the $S_{11}$ scattering amplitudes of the $\pi N$ channel up to the energy $\sqrt{s} \sim 1400 \mathrm{MeV}$. We show the calculated scattering amplitudes of the $S_{11} \pi N$ channel in Fig. 3 for the following three cases: (A) $a=0.53$ without $\mathrm{SU}(3)$ breaking, (B) $a=1.33$ with $\mathrm{SU}(3)$ symmetry breaking and (C) $a=2.24$ with physical meson decay constants. In all cases, the scattering amplitudes and cross sections (we do not show the cross sections here) are not well reproduced. The results of (A) seems to have some struc-

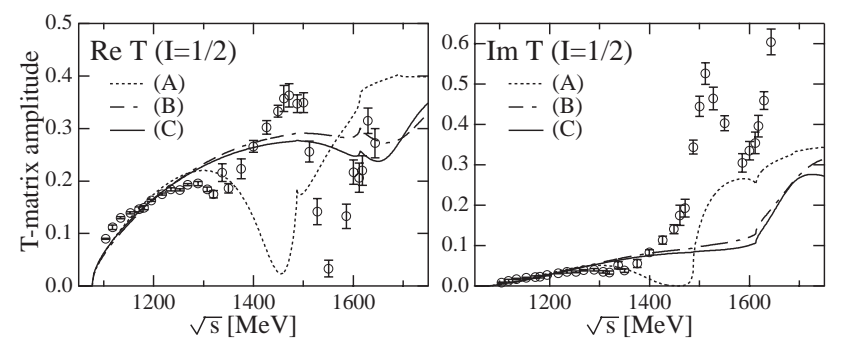

FIG. 3: Real and imaginary parts of the $S_{11}$ T-matrix amplitudes of $\pi N \rightarrow \pi N$. Dotted lines show the results with $a=0.53(\mathrm{~A})$, dash-dotted lines show the results including the $\mathrm{SU}(3)$ breaking interaction with $a=1.33(\mathrm{~B})$, and solid lines show the results including the $\mathrm{SU}(3)$ breaking and the physical $f$ with $a=2.24$ (C). Open circles with error bars are experimental data taken from Refs. [26].

ture around the $N(1535)$ energies, but it is far from the observed amplitude. Reasonable agreement with data is achieved only when channel dependent subtraction constants are introduced as shown in Table I 6].

In this work, motivated by the channel dependence of the parameters and symmetry consideration, we have tried to reproduce the observed cross sections and the resonance properties using a single subtraction constant. In the $S=-1$ channel, without the symmetry breaking terms, $a=-1.96$ is determined by the threshold branching ratios of the $K^{-} p$ scatterings. With this parameter (A), the total cross sections of the $K^{-} p$ scatterings are reproduced well, as well as the mass distribution for $\Lambda(1405)$ is. (See Figs 1 and 2) This value is closed to the $a \sim-2$ corresponds to $\Lambda=630 \mathrm{MeV}$ in the threemomentum cut-off reguralization [8]. The elementary interaction of the $\bar{K} N$ system is sufficiently attractive, and a resummation of the coupled channel interactions generates the $\Lambda(1405)$ resonance at the correct position, by imposing the unitarity condition with the natural value for the cut-off parameter. Hence the wave function of $\Lambda(1405)$ is largely dominated by the $\bar{K} N$ component.

On the other hand, in the $S=0$ channel, if one uses the natural value for the subtraction constant as in the $S=-1$ channel, the attraction of the meson-baryon interaction becomes so strong that an unexpected resonance is generated at around $\sqrt{s} \simeq 1250 \mathrm{MeV}$. Therefore, repulsive component is necessary to reproduce the observed $\pi N$ scattering. However, with the fitted value $a \sim 0.5$, the $N(1535)$ resonance is not generated.

From the above observation, we see that the unitarized amplitudes are very sensitive to the attractive component of the interaction. Even including the SU(3) breaking terms, the interaction derived from the chiral Lagrangian alone do not describe all scattering amplitudes simultaneously. Both the fundamental interaction and the subtraction constants are important in order to reproduce proper results. For smaller $a$, the interaction becomes more attractive, and for larger $a$, less attractive. For $S=0$, we need to choose $a \sim 0.5$ in order to suppress 
the attraction from the $\pi N$ interaction in contrast with the natural value $a \sim-2$ in the $S=-1$ channel. Therefore, it is not possible to reproduce both the $\Lambda(1405)$ resonance properties and the low energy $\pi N$ scattering with a common subtraction constant.

At this point, it is useful to discuss slightly in detail the structure of the $\Lambda(1405)$ resonance. Although the properties of $\Lambda(1405)$ has not been reproduced well with the $\mathrm{SU}(3)$ breaking terms as shown in the $\pi \Sigma$ mass distribution (Fig 2), we still have found the two poles for $\Lambda(1405)$ in the scattering amplitudes in the second Riemann sheet. The property of the two poles are investigated recently in detail and is related to the $\mathrm{SU}(3)$ structure of the meson and baryon states $8,[11,27,28]$. In the present study, we find $z_{1}(B)=1424+1.6 i$ and $z_{2}(B)=1389+135 i$ for the parameter (B). The pole $z_{1}$, which is located very close to the real axis, is responsible for the sharp peak. When the $\mathrm{SU}(3)$ breaking of the meson decay constants is introduced (C), the poles are $z_{1}(C)=1424+2.6 i$ and $z_{2}(C)=1363+87 i$, where $z_{1}$ is still close to the real axis, while $z_{2}$ moves significantly.

The shape of the $\pi \Sigma$ mass distribution is strongly influenced by the location of the poles. In this case, the poles $z_{2}$ is sensitive to the pion decay constant. Since the resonance of $z_{2}(B)$ has a strong coupling to the $\pi \Sigma$ channel [1], the resonance properties are very much affected by the $\pi \Sigma$ interaction. In the chiral Lagrangian, the interaction is attractive as in the Weinberg-Tomozawa term, which contains a coupling strength proportional to the inverse square of the pion decay constant. Therefore, by changing the decay constant from the $\mathrm{SU}(3)$ averaged value $(107 \mathrm{MeV}$, case B) to the physical value $(93 \mathrm{MeV}$, case $\mathrm{C}$ ), the strength of the attractive $\pi \Sigma$ interaction is enhanced by $\sim 30 \%$. This shifts the real part of $z_{2}$ to the lower side. At the same time this reduces the phase space and hence the imaginary part decreases.

To summarize shortly, we have studied the flavor SU(3) symmetry breaking effect in the meson-baryon scatterings in the chiral unitary model. A reasonable prescription from symmetry consideration by including the symmetry breaking mass terms, which appear in the nextto-leading order of the chiral expansion, make theoretical predictions worse. So far, except for the use of channel dependent subtraction constants, we do not know what would resolve this problem. In the present framework, the role of the subtraction constants is very important.

A better understanding may be provided by introducing genuine resonance components. Very naively such states could be quark originated as expected from the success of the quark model for baryon resonances. Full coupled channel studies of meson-baryon and quark degrees of freedom would be useful in order to resolve the problem discussed in the present study. Such an analysis will provide more microscopic understanding for the resonance structure.

\section{Acknowledgments}

We would like to thank Profs. E. Oset, H. -Ch. Kim and $\mathrm{W}$. Weise for useful discussions.
[1] N. Kaiser, P. B. Siegel, and W. Weise, Phys. Lett. B362, 23 (1995).

[2] B. Krippa, Phys. Rev. C58, 1333 (1998).

[3] E. Oset and A. Ramos, Nucl. Phys. A635, 99 (1998).

[4] M. F. M. Lutz and E. E. Kolomeitsev, Nucl. Phys. A700, 193 (2002).

[5] E. Oset, A. Ramos, and C. Bennhold, Phys. Lett. B527, 99 (2002).

[6] T. Inoue, E. Oset, and M. J. Vicente Vacas, Phys. Rev. C65, 035204 (2002).

[7] A. Ramos, E. Oset and C. Bennhold, Phys. Rev. Lett. 89, 252001 (2002)

[8] J. A. Oller and U. G. Meissner, Phys. Lett. B500, 263 (2001).

[9] T. Hyodo, S. I. Nam, D. Jido, and A. Hosaka, in preparation

[10] D. Jido, E. Oset and A. Ramos, Phys. Rev. C 66, 055203 (2002)

[11] D. Jido, J. A. Oller, E. Oset, A. Ramos, and U. G. Meissner, nucl-th/0303062

[12] For example, J. F. Donoghue, E. Golowich, and B. R. Holstein, Dynamics of the standard model (Cambridge University Press, London, 1992).

[13] M. Gell-Mann, R. J. Oakes, and B. Renner, Phys. Rev. 175, 2195 (1968).

[14] R. J. Nowak et al., Nucl. Phys. B139, 61 (1978).

[15] D. N. Tovee et al., Nucl. Phys. B33, 493 (1971).
[16] T. S. Mast, M. Alston-Garnjost, R. O. Bangerter, A. S. Barbaro-Galtieri, F. T. Solmitz, and R. D. Tripp, Phys. Rev. D14, 13 (1976).

[17] J. Ciborowski et al., J. Phys. G8, 13 (1982).

[18] R. O. Bangerter, M. Alston-Garnjost, A. S. BarbaroGaltieri, T. S. Mast, F. T. Solmitz, and R. D. Tripp, Phys. Rev. D23, 1484 (1981).

[19] T. S. Mast, M. Alston-Garnjost, R. O. Bangerter, A. S. Barbaro-Galtieri, F. T. Solmitz, and R. D. Tripp, Phys. Rev. D11, 3078 (1975).

[20] M. Sakitt, T. B. Day, R. G. Glasser, N. Seeman, J. H. Friedman, W. E. Humphrey, and R. R. Ross, Phys. Rev. 139, B719 (1965).

[21] M. Csejthey-Barth et al., Phys. Lett. 16, 89 (1965).

[22] J. K. Kim, Phys. Rev. Lett. 14, 29 (1965); Columbia University Report, Nevis 149 (1966).

[23] W. Kittel, G. Ptter, and I. Wacek, Phys. Lett. 21, 349 (1966).

[24] H. Göing, Nuovo Cim. 16, 848 (1960).

[25] R. J. Hemingway, Nucl. Phys. B253, 742 (1985).

[26] Center of Nuclear Study, http://gwdac.phys.gwu.edu .

[27] D. Jido, A. Hosaka, J. C. Nacher, E. Oset, and A. Ramos, Phys. Rev. C66, 025203 (2002).

[28] C. Garcia-Recio, J. Nieves, E. Ruiz Arriola, and M. J. Vicente Vacas, hep-ph/0210311 